\title{
Screening for head and neck second primary tumors in patients with esophageal squamous cell cancer: A systematic review and meta-analysis
}

United European Gastroenterology Journal 2019, Vol. 7(10) 1304-1311 (C) Author(s) 2019 (c) (i) (2) Article reuse guidelines: sagepub.com/journals-permissions DOI: $10.1177 / 2050640619856459$ journals.sagepub.com/home/ueg @SAGE

\author{
SEM van de $\operatorname{Ven}^{1}{ }^{D}, 0$ Bugter $^{2}$, JA Hardillo $^{2}$, MJ Bruno $^{1}$, \\ RJ Baatenburg de Jong ${ }^{2}$ and AD Koch $^{1}$
}

\begin{abstract}
Background: Esophageal squamous cell carcinomas (ESCCs) are often accompanied by head and neck second primary tumors (HNSPTs). The prognosis of patients with an additional HNSPT is worse compared with patients with only ESCC. Therefore, early detection of HNSPTs may improve the overall outcome of patients with ESCC.

The purpose of this study was to investigate the yield of endoscopic screening for HNSPTs in patients with primary ESCC. Methods: We conducted a systematic literature search of all available databases. Studies were included if ESCC patients were endoscopically screened for HNSPT. The primary outcome was the pooled prevalence of HNSPTs.

Results: Twelve studies, all performed in Japan, were included in this systematic review with a total of 6483 patients. The pooled prevalence of HNSPTs was $6.7 \%$ (95\% confidence interval: 4.9-8.4). The overall heterogeneity was high across the studies $\left(I^{2}=89.0 \%, p<0.001\right)$. Most HNSPTs were low stage $(85.3 \%)$ and located in the hypopharynx $(60.3 \%)$. The proportion of synchronous (48.2\%) and metachronous $(51.8 \%)$ HNSPTs was comparable.

Conclusion: Based on our results, HNSPT screening could be considered in patients with primary ESCC. All studies were performed in Japan; it is therefore not clear whether this consideration applies to the Western world.
\end{abstract}

Keywords

Cancer, endoscopy, esophagus, oncology, screening

Received: 29 March 2019; accepted: 20 May 2019

\section{Key summary}

- The prognosis of patients with esophageal squamous cell carcinoma (ESCC) and an additional head and neck second primary tumor (HNSPT) is worse than patients with only ESCC. Early detection of HNSPT may improve patient outcome.

- The pooled prevalence of HNSPT in patients with ESCC is $6.7 \%$.

- Most HNSPTs are low stage $(85.3 \%)$ and located in the hypopharynx $(60.3 \%)$.

- HNSPT screening could be considered in patients with primary ESCC.

- Western screening studies are needed, since all studies have been performed in Japan.

\section{Introduction}

Both esophageal and head and neck $(\mathrm{HN})$ cancer are common malignancies worldwide. ${ }^{1,2}$ Esophageal squamous cell carcinoma (ESCC) is the most common histologic type in the esophagus. ${ }^{3}$ Patients with ESCC frequently develop second primary tumors (SPTs) in the upper aerodigestive tract, most often in the $\mathrm{HN}$
${ }^{1}$ Department of Gastroenterology and Hepatology, Erasmus MC Cancer Institute, Rotterdam, the Netherlands

${ }^{2}$ Department of Otorhinolaryngology and Head and Neck Surgery, Erasmus MC Cancer Institute, Rotterdam, the Netherlands

Corresponding author:

Arjun D. Koch, Erasmus MC Cancer Institute, Department of Gastroenterology and Hepatology, Postbox 2040, 3000 CA, Rotterdam, the Netherlands.

Email: a.d.koch@erasmusmc.nl 
region, but also in the esophagus and lungs. ${ }^{4,5}$ The presence of SPTs can be explained by the "field cancerization" theory: Premalignant epithelial changes can occur because of chronic local exposure to common carcinogens, such as alcohol and tobacco, which contributes to the development of syn- and metachronous SPTs. ${ }^{6}$ An important risk factor in Western countries for the development both of ESCC and SPTs is alcohol. ${ }^{7,8}$

Head and neck second primary tumors (HNSPTs) in patients with primary ESCC are reported to be up to $7 \%$ in retrospective studies. ${ }^{4,5}$ The prognosis and survival of patients with esophageal cancer (EC) is poor because most ECs are diagnosed in advanced stages, when definitive cure is most often not achievable. ${ }^{9}$ The long-term prognosis is even worse in patients with an additional HNSPT compared with ESCC alone (five-year survival rate of $9.2 \%$ vs $21.0 \%$ ). ${ }^{10}$ This poor prognosis makes early detection of HNSPTs vitally important, especially for ESCC patients with lowstage tumors that could be treated endoscopically, since they have a considerably higher five-year survival rate. ${ }^{11}$

Different endoscopic techniques for $\mathrm{HN}$ cancer screening have been studied. Although Lugol chromoendoscopy is often used in the esophagus to detect dysplastic mucosal lesions, it is known to cause side effects in the HN region such as chest pain and aspiration. ${ }^{12}$ Narrow-band imaging (NBI) seems to be the best technique for the detection of HNSPTs in patients with primary ESCC. ${ }^{13}$ The HNSPT detection rate is significantly higher using NBI (sensitivity 100\%, specificity 97.5\%) compared with only white-light endoscopy (WL). ${ }^{13}$ The sensitivity of fluorodeoxyglucose-positron-emission tomography/ computed tomography (CT) for the detection of HNSPTs is $61.5 \%$; more HNSPTs have been detected by endoscopy. ${ }^{14}$

The European Society for Medical Oncology Clinical Practice Guidelines for EC recommends endoscopic screening of the $\mathrm{HN}$ region and tracheabronchoscopy to detect SPTs in the upper aerodigestive tract in all ESCC patients with chronic tobacco and alcohol consumption. ${ }^{15}$ However, no Western screening studies have been published to date. The Japanese EC guidelines recommend appropriate diagnostic measures of other organs (HN, stomach, large intestine) after treatment of ESCC because of the risk of developing SPTs. ${ }^{16}$ However, no specific screening recommendations (i.e. diagnostic method and time of screening) are mentioned. ${ }^{16}$

We have performed a systematic review and metaanalysis of studies that investigated the use of endoscopic screening for the detection of HNSPTs in patients with primary ESCC. Our primary objective was to investigate the yield of endoscopic screening for HNSPTs in patients with primary ESCC. Our secondary objectives were to investigate whether there is evidence to justify endoscopic HN screening in primary ESCC patients in the Western world, and to investigate whether screening should be performed synchronously or metachronously.

\section{Materials and methods}

\section{Literature search and selection criteria}

A systematic literature search was performed in collaboration with the medical library of the Erasmus University Rotterdam, the Netherlands, in February 2019, with no limit on publication date. The search was performed in PubMed, Embase, Medline, Cochrane Central, Google Scholar and Web of Science databases. The full electronic search strategy for the Embase database is provided in Supplementary Appendix 1. The search was limited to English studies performed on humans. After removing duplicate citations, the remaining articles were reviewed based on title and abstract by two independent reviewers (S.E.M.V. and O.B.). Subsequently, the full text of the remaining articles was screened by the same authors and discrepancies were discussed mutually. If there was no agreement, a third party was involved (A.D.K.). Studies were included if patients with primary ESCC were endoscopically screened for HNSPTs. Studies were excluded if patients with primary head and neck squamous cell carcinoma (HNSCC) were screened for esophageal SPTs, since we investigated the yield of HNSPT screening in patients with primary ESCC. Moreover, these studies are already included in a systematic review about screening for esophageal SPT in patients with primary HNSCC. ${ }^{17}$ Studies without full text, case reports, reviews, and studies in which only imaging techniques were used to detect HNSPTs were excluded. References of the retrieved studies were manually screened to locate additional studies.

\section{Study quality}

The Methodological Index for Non-Randomized Studies (MINORS) was used to test the risk of bias and the methodological quality of the selected studies. ${ }^{18}$ The study relevance was determined using a checklist. This checklist includes (1) the impact factor of the publishing journal (indication of peer-review quality), (2) data of the HNSPT sublocation and (3) text clarity (Table 1). The total quality score of the studies was the sum of the MINORS and relevance criteria score. The total scores were classified as low ( $\leq 10$ points), 
Table 1. Relevance criteria.

\begin{tabular}{llll}
\hline & \multicolumn{2}{l}{ Score } & \\
\cline { 2 - 4 } Criteria & 0 & 1 & 2 \\
\hline Text clarity & Low & Medium & High \\
Sublocation & No & - & Yes \\
Impact factor & $<2$ & $2-3.9$ & $\geq 4$ \\
\hline
\end{tabular}

medium (11-14 points) or high ( $\geq 15$ points). Studies classified as medium and high were included.

\section{Data extraction and outcome parameters}

Data from included studies were summarized as a Preferred Reporting Items for Systematic Reviews and Meta-Analyses checklist and flowchart. ${ }^{19}$ The primary outcome was the prevalence of diagnosed HNSPTs. An HNSPT was defined as a lesion in the $\mathrm{HN}$ region classified as carcinoma in situ or carcinoma. With NBI, these lesions can be described as welldemarcated brownish areas without magnification, irregular microvascular patterns, and increased intraepithelial papillary capillary loops. ${ }^{20}$ Secondary outcomes were recorded when possible: (1) HNSPT prevalence per sublocation (upper, middle and lower esophagus) and per tumor stage (0 to IV according to the Vienna classification of gastrointestinal epithelial neoplasia) of the primary ESCC, (2) synchronously ( $\leq 6$ months after diagnosis of primary ESCC) or metachronously ( $>6$ months after diagnosis of primary ESCC) diagnosed HNSPTs and (3) tumor stage and sublocation of HNSPTs. ${ }^{21}$ Other characteristics of the studies were also recorded: first author, publication year, study design, size and country of the study population.

\section{Statistical analysis}

For each study, the HNSPT prevalence was calculated (total number of HNSPTs divided by the total population that was screened). The standard error (SE) was calculated from the prevalence using the following formula: $\quad \mathrm{SE}=\sqrt{(p \times(1-p)) / n}, \quad p=$ prevalence and $n=$ total number of patients with ESCC that were screened. Estimation of the prevalence was carried out with the aid of a random-effects meta-analysis. Combined estimates and 95\% confidence intervals (CIs) for the HNSPT prevalence rates were calculated. The heterogeneity among studies was measured by calculating the inconsistency index $\left(I^{2}\right)$, with values from $0 \%$ to $100 \%$ (maximum heterogeneity). Categories of low, moderate and high were assigned to $I^{2}$ values of $25 \%, 50 \%$ and $75 \%$, respectively. ${ }^{22}$

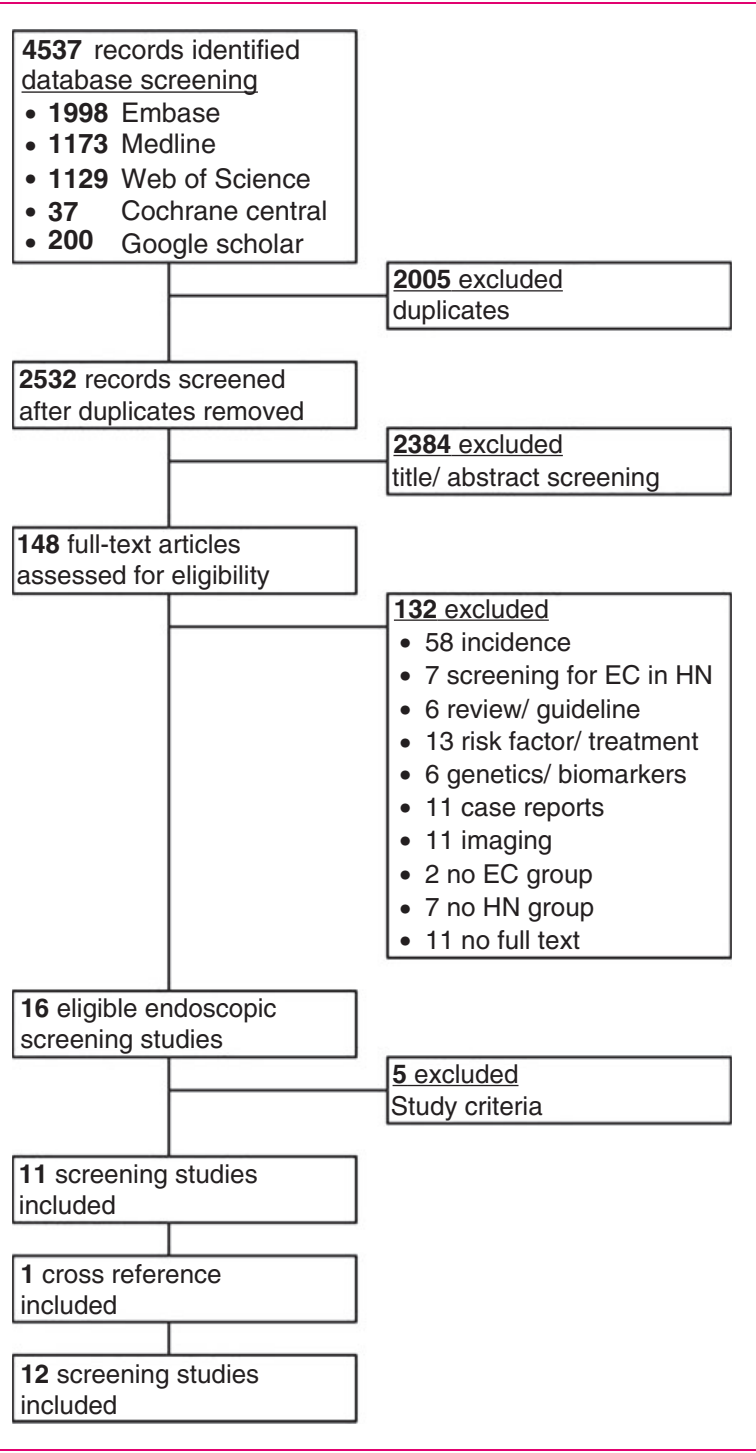

Figure 1. Study selection process.

EC: esophageal cancer; HN: head and neck cancer.

When $I^{2} \geq 50 \%$, there was evidence of moderate or high heterogeneity.

\section{Results}

\section{Study selection and quality assessment}

The study selection process and eligibility assessment are outlined in Figure 1. The literature search identified 4537 citations. After screening, 148 articles were examined by full-text review for their eligibility by two reviewers (S.E.M.V. and O.B.). Discrepancies were discussed mutually without any final disagreements. One additional study was included after screening the references. Twelve studies were included in our systematic review. ${ }^{7,10,13,20,23-30}$ Exclusion reasons are shown in 
Table 2. Study characteristics and quality score of all 12 studies.

\begin{tabular}{|c|c|c|c|c|c|c|c|c|c|}
\hline \multirow[b]{2}{*}{ Authors ${ }^{\text {Ref }}$} & \multirow[b]{2}{*}{ Year } & \multirow[b]{2}{*}{ Design } & \multirow[b]{2}{*}{$N$} & \multirow[b]{2}{*}{ Method } & \multicolumn{3}{|c|}{ Quality Score } & \multirow[b]{2}{*}{ Quality } & \multirow[b]{2}{*}{ Screening sites } \\
\hline & & & & & MINORS & Rel & Total & & \\
\hline Abiko et al. ${ }^{7}$ & 2018 & Pro & 158 & WL & 18 & 3 & 21 & High & Larynx \\
\hline Onochi et al. ${ }^{29}$ & 2018 & Retro & 285 & WL & 10 & 3 & 13 & Medium & Oro-, hypopharynx \\
\hline Morimoto et al. ${ }^{23}$ & 2017 & Retro & 307 & $W L+N B I$ & 18 & 5 & 23 & High & $\begin{array}{l}\text { Oro-, hypopharynx } \\
\text { Larynx }\end{array}$ \\
\hline Kaneko et al. ${ }^{26}$ & 2013 & Retro & 348 & $\mathrm{WL}+\mathrm{NBI}$ & 9 & 4 & 13 & Medium & Oral cavity \\
\hline Katada et al. ${ }^{30}$ & 2012 & Pro & 71 & $\mathrm{WL}+\mathrm{NBI}$ & 16 & 5 & 21 & High & $\begin{array}{l}\text { Pharynx } \\
\text { Head and neck region }{ }^{a}\end{array}$ \\
\hline Muto et al. ${ }^{20}$ & 2010 & Pro & 320 & $\mathrm{WL}+\mathrm{NBI}$ & 23 & 4 & 27 & High & Oro-, hypopharynx \\
\hline Nonaka et al. ${ }^{13}$ & 2009 & Pro & 424 & $\mathrm{WL}+\mathrm{NBI}$ & 19 & 5 & 24 & High & Pharynx \\
\hline Lo et al. ${ }^{10}$ & 2008 & Pro & 1675 & WL & 18 & 3 & 21 & High & Head neck region ${ }^{a}$ \\
\hline Watanabe et al. ${ }^{28}$ & 2007 & Pro & 1118 & Lugol & 10 & 3 & 13 & Medium & Head neck region ${ }^{a}$ \\
\hline Shimizu et al. ${ }^{24}$ & 2003 & Pro & 99 & Lugol & 18 & 5 & 22 & High & Hypopharynx, larynx \\
\hline Kagei et al. ${ }^{27}$ & 2002 & Pro & 1479 & WL & 10 & 2 & 12 & Medium & Head neck region ${ }^{a}$ \\
\hline Motoyama et al. ${ }^{25}$ & 2003 & Pro & 200 & WL & 13 & 4 & 17 & High & Larynx \\
\hline
\end{tabular}

MINORS: Methodological Index for Non-Randomized Studies; NBI: narrow-band imaging; Pro: prospective; Rel: relevance; Retro: retrospective; WL: whitelight endoscopy.

${ }^{a}$ Nasal cavity, oral cavity, naso-, oro-, and hypopharynx and larynx.

Figure 1. All 12 included studies qualified as medium or high (Table 2). The relevance criteria score ranged between 0 and 5 points (maximum possible score is 6). The MINORS criteria score ranged from 9 to 23 points (maximum possible score of 24).

\section{Study characteristics}

The study characteristics are reported in Table 2. All studies were performed in Japan and published between 2002 and 2018. Nine of the 12 studies $(75 \%)$ collected their data prospectively $7,10,13,20,24,25,27,28,30$ and three $(25 \%)$ retrospectively. ${ }^{23,26,29}$ The total number of included patients was 6483 (median, 313; range, 71-1674). In two studies Lugol iodine was used for screening of the HN region. ${ }^{24,28}$ In five other studies both WL and NBI were used for screening. ${ }^{13,20,23,26,30}$ In the remaining five studies, only WL was used for screening. ${ }^{7,10,25,27,29}$ In only four studies, the entire HN region was screened. ${ }^{10,20,27,28,30}$ Screening was limited to the pharynx and larynx, sublocations known to be at an increased risk, in most other studies. Eleven of the 12 studies screened only patients with ESCC. ${ }^{7,10,13,20,23-26,28-30}$ One study screened patients with both ESCC (93\%) and esophageal adenocarcinoma $(7 \%) .{ }^{27}$ In total, $98 \%$ of the esophageal tumors were squamous cell carcinomas and $2 \%$ adenocarcinomas. Screening was performed by an otolaryngologist or head and neck surgeon in 5/12 included studies. $^{7,24,25,27,28}$ Screening was performed by a gastroenterologist in $2 / 12$ studies. $^{20,29}$ In these two studies, only the oropharynx and hypopharynx were screened. In 5/12 included studies, however, it was not clearly reported who performed the screening endoscopy of the head and neck region (otolaryngologist or gastroenterologist). ${ }^{10,13,23,26,30}$

\section{Pooled SPT prevalence}

The prevalence of HNSPTs in patients with ESCC is shown for each study in Figure 2. In total, 353/6483 patients were diagnosed with HNSPT. Meta-analysis with a random-effect model was used to calculate the pooled prevalence since the $I^{2}$ was $89.0 \%$. The pooled prevalence for HNSPTs of the 12 included studies was 6.7\% (95\% CI: $4.9-8.4 \%$ ) (Figure 2).

\section{Sublocation of HNSPT and tumor stage}

The sublocation of the HNSPTs was reported in eight of the 12 studies, for a total of 288 SPTs. ${ }^{10,13,23-26,28,30} \mathrm{In}$ one study the sublocation was reported together for primary HN tumors and HNSPTs. Therefore, we excluded the study for this subanalysis. ${ }^{10}$ A total of 234 HNSPTs remained. The combined data showed that $60 \%(141 /$ 234) of all HNSPTs were located in the hypopharynx, $18 \%(41 / 234)$ in the oropharynx, $11 \%(26 / 234)$ in the oral cavity, $9 \%(22 / 234)$ in the larynx and $2 \%(4 / 234)$ in other sublocations. In total, 405 HNSPTs were detected in 353 patients. Tumor stage of HNSPTs was reported in eight of the 12 studies. $^{13,20,23,24,26-28,30}$ Morimoto et al. reported tumor characteristics of metachronous 


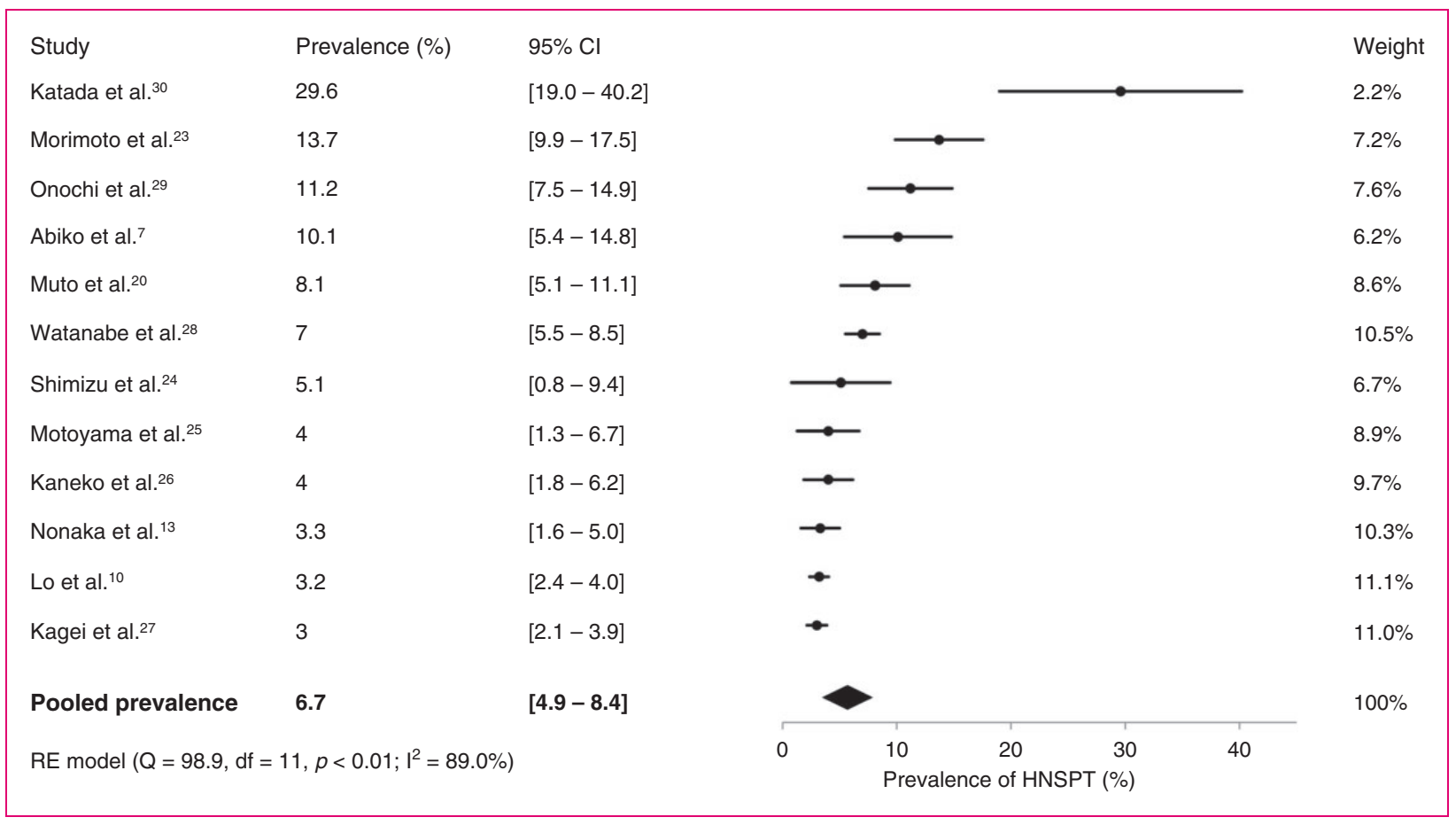

Figure 2. Forest plot of prevalence of head and neck second primary tumors in patients with esophageal squamous cell carcinoma. $\mathrm{Cl}$ : confidence interval; df: degree of freedom; HNSPT: head and neck second primary tumor; $l^{2}$ : inconsistency index; RE: random-effects.

HNSPTs only. ${ }^{23}$ Combined data showed that tumor stage was available for $62 \%$ of the HNSPTs $(251 / 405)$. Overall, HNSPTs were classified as low stage (stage 0, I and II) in $85 \%(214 / 251)$ and high stage (stage III and IV) in $15 \%(37 / 251)$.

\section{Time to diagnosis}

Eight studies performed both syn- and metachronous endoscopic screening of the HN region, ${ }^{10,20,23-25,28-30}$ and six studies adequately reported the percentage of detected synchronous and metachronous HNSPTs (Table 3). ${ }^{10,23-25,28,29}$ The median time to metachronous HNSPT diagnosis of these six studies ranged from 12 to 48 months. The time to SPT diagnosis in ESCC patients was reported for all SPTs together in Motoyama et al., not separately for HNSPTs. ${ }^{25}$

Two studies performed only HN screening synchronously, ${ }^{26,27}$ and two only metachronously. ${ }^{7,13}$ The HNSPT prevalence in the study by Nonaka and colleagues ${ }^{13}$ was $3.3 \%(14 / 424)$ with a median detection period of 27.6 months (range, 7.1-143.5) in patients screened with NBI and 101.0 months (range, 11.0 134.5) in patients screened with WL.

\section{Primary ESCC tumor characteristics}

Only four studies reported the sublocation of the index esophageal tumor. ${ }^{10,24,25,27}$ One study included only patients who underwent esophagectomy for thoracic ESCC. ${ }^{25}$ The prevalence of HNSPTs in this study was $4.0 \%$. The average percentages of index upper, middle and lower ESCC of the other three studies were $17.0 \%$, $57.7 \%$ and $25.3 \%$ respectively. ${ }^{10,24,27}$ However, they did not report the prevalence of HNSPT per ESCC sublocation. The tumor stage of the primary ESCC was reported in nine studies $(75 \%) .7,10,20,23-25,27,29,30$ On average, most esophageal lesions were stage 1 $(29.0 \%)$ and stage $3(29.8 \%)$. Other tumor stages were 0 (high-grade dysplasia) (7.3\%), $2(20.2 \%)$ and stage $4(13.6 \%)$. The HNSPT prevalence per tumor stage of the primary ESCC was reported in three studies, for which only superficial ESCCs (stage 0 and I) were screened. ${ }^{7,29,30}$

\section{Discussion}

To our knowledge, this is the first systematic review on endoscopic screening for HNSPTs in patients with primary ESCC. Worldwide, the incidence of $\mathrm{HN}$ cancer is more than 550,000 cases annually. ${ }^{2}$ We found an HNSPT (pooled) prevalence of $6.7 \%$. Most HNSPTs were located in the hypopharynx $(60.3 \%)$ and classified as low stage $(85.3 \%)$. The proportion of synchronous and metachronous HNSPTs was comparable. Although the worldwide incidence cannot be compared directly with the pooled prevalence from this metaanalysis, the concept of endoscopic screening in 
Table 3. Percentages of synchronous and metachronous HNSPT.

\begin{tabular}{|c|c|c|c|c|}
\hline Authors ${ }^{\text {Ref }}$ & Total SPTs & $\begin{array}{l}\text { Synchronous } \\
\text { HNSPTs (\%) }\end{array}$ & $\begin{array}{l}\text { Metachronous } \\
\text { HNSPTs (\%) }\end{array}$ & $\begin{array}{l}\text { Median time to diagnosis, } \\
\text { months (range) }\end{array}$ \\
\hline Morimoto et al. ${ }^{23}$ & 67 & $14(21 \%)$ & $53(79 \%)$ & $31(7-107)$ \\
\hline Shimizu et al. ${ }^{24}$ & 5 & 0 & $5(100 \%)$ & $37(15-61)$ \\
\hline Motoyama et al. ${ }^{25}$ & 8 & 0 & $8(100 \%)$ & Not reported \\
\hline Watanabe et al. ${ }^{28}$ & 85 & $37(44 \%)$ & $48(56 \%)$ & $48(12-103)$ \\
\hline Onochi et al. ${ }^{29}$ & 32 & $23(72 \%)$ & $9(28 \%)$ & Not reported \\
\hline Lo et al. ${ }^{10}$ & 54 & $47(87 \%)$ & $7(13 \%)$ & $12(8-110)$ \\
\hline Total & 251 & $121(48 \%)$ & $130(52 \%)$ & \\
\hline
\end{tabular}

HNSPT: head and neck second primary tumor; SPT: second primary tumor.

patients with ESCC shows promise. An increase in early detection of HNSPTs could potentially improve the overall survival of ESCC patients.

Screening in Western countries will possibly show a different HNSPT prevalence because the etiology partly differs among these continents, and ESCC and HNSCC have a higher prevalence in Asia. ${ }^{3,31}$ The etiology of ESCC in Asia is, besides smoking and alcohol intake, clearly linked to a lowered fruit and vegetable intake. ${ }^{32}$ The overall incidence of $\mathrm{HN}$ cancer in Japan was increasing, whereas the incidence in the United States was decreasing. ${ }^{31,33}$ Since the included studies were performed in Japan, it is unlikely that these results can be applied to the contemporary Western population.

Nonscreening Asian studies have reported HNSPT prevalence up to $7 \%$ in patients with primary ESCC. ${ }^{4,5}$ This is lower than the prevalence of the included studies $(3.0 \%-29.6 \%)$. This might indicate that active screening of ESCC patients increases the number of detected HNSPTs. ${ }^{23}$ Early diagnosis and treatment of both tumors can increase survival rate. ${ }^{23,34}$

Eighty-five percent of the HNSPTs were classified as low stage, which is higher than in the general HN cancer population. ${ }^{35}$ Morimoto et al. reported a higher percentage of low-stage HNSPTs in patients with primary ESCC who were actively screened, and $83 \%$ of these HNSPTs could be treated with endoscopic resection. ${ }^{23}$ Furthermore, survival was better in ESCC patients with HNSPTs who were actively screened. ${ }^{23}$ ESCC patients could benefit from HN screening because this could result in an increased detection of superficial HN cancer, which can be treated with curative intent.

There is a lack of standardization in $\mathrm{HN}$ examination protocols among the included studies because different screening techniques are used (WL, NBI and Lugol). Studies that compared NBI with WL described a significantly higher detection rate of HNSPTs and a higher sensitivity and accuracy when using NBI. ${ }^{13,20,23,26}$ It would therefore be useful to always perform HN screening with WL and NBI. Lugol chromoendoscopy is not recommended in the $\mathrm{HN}$ region because this has to be performed under general anesthesia because of possible side effects. ${ }^{28}$

The average percentage of synchronous and metachronous HNSPTs of all studies together is comparable. This could indicate that HN screening in patients with ESCC should be performed during work-up and followup. The median detection time of metachronous HNSPTs ranged from 12 to 101 months. ${ }^{10,13,23,24,28}$ However, the optimal moment for screening during follow-up has yet to be defined.

Our systematic review showed that $78 \%$ of the HNSPTs were located in the pharynx, which suggests that the pharynx has the highest risk of developing SPTs. Moreover, patients with pharyngeal cancer also showed the highest prevalence of esophageal SPTs. ${ }^{17}$ The pharynx is the head and neck region that should definitely be screened in patients with primary ESCC. Although 10 of the 12 included studies performed screening of the pharynx, only four studies screened the whole $\mathrm{HN}$ region. We are aware of the fact that, of these four studies, only two studies reported the HNSPT sublocation. ${ }^{28,30}$ It was not possible to state whether there was a correlation between ESCC tumor stage and the occurrence of HNSPTs since this information was reported in only three studies. ${ }^{7,29,30}$ In these studies, only superficial ESCCs (stage 0 and I) were screened, which could underestimate the true HNSPT prevalence per ESCC tumor stage.

Some potential limitations about the methodology of the included studies need to be discussed: 1) Different screening techniques (i.e. WL, NBI, Lugol chromoendoscopy) were used. The combination of WL and NBI has the highest HNSPT detection rate; potential HNSPTs could be missed when using only WL; 2) one study performed screening with endoscopy and CT scan. ${ }^{27}$ It was not clearly described which proportion of HNSPTs were detected by endoscopic screening. The proportion of HNSPTs detected by endoscopic screening could be lower than reported; 3) a different definition of metachronous and synchronous 
was used in three studies, whereby the comparison of the different studies was more difficult and the proportion of metachronous SPTs could be higher than reported; ${ }^{7,27,28} 4$ ) only four studies screened the whole $\mathrm{HN}$ region. Therefore, we could not easily determine which HN sublocation was at increased risk of developing HNSPTs; and 5) because this meta-analysis contained both prospective and retrospective data, a significant bias may be present.

In conclusion, the pooled prevalence of HNSPTs in patients with primary ESCC is $6.7 \%$. Most HNSPTs were classified as low stage. Patients with low-stage HN tumors can be treated curatively with an excellent prognosis. Screening for HNSPTs could therefore be useful in ESCC patients. More screening studies are needed to investigate which type of ESCC (i.e. tumor stage and sublocation) increases the risk of HNSPTs and to report on risk factors associated with HNSPTs. More important, it is necessary to perform Western screening studies to assess the HNSPT prevalence since it is unclear whether the results of Asian studies can be extrapolated to the Western population. Head and neck examination protocols should be standardized in Japan; screening should be performed during work-up and follow-up with WL in combination with NBI. The pharynx is the head and neck region that should always be screened.

\section{Acknowledgment}

The authors thank W.M. Bramer of the Erasmus MC medical library for developing the search strategy.

\section{Declaration of conflicting interests}

None declared.

\section{Funding}

This research received no specific grant from any funding agency in the public, commercial, or not-for-profit sectors.

\section{Ethics approval}

No ethical approval was required, since this is a systematic review.

\section{Informed consent}

Informed consent was not required for this study.

\section{ORCID ID}

SEM van de Ven (D) https://orcid.org/0000-0001-9793-0957

\section{References}

1. Ferlay J, Soerjomataram I, Dikshit R, et al. Cancer incidence and mortality worldwide: Sources, methods and major patterns in GLOBOCAN 2012. Int J Cancer 2015; 136: E359-E386.
2. Global Burden of Disease Cancer Collaboration, Fitzmaurice C, Allen C, et al. Global, regional, and national cancer incidence, mortality, years of life lost, years lived with disability, and disability-adjusted lifeyears for 32 cancer groups, 1990 to 2015: A systematic analysis for the Global Burden of Disease Study. JAMA Oncol 2017; 3: 524-548.

3. Arnold M, Soerjomataram I, Ferlay J, et al. Global incidence of oesophageal cancer by histological subtype in 2012. Gut 2015; 64: 381-387.

4. Poon RT, Law SY, Chu KM, et al. Multiple primary cancers in esophageal squamous cell carcinoma: Incidence and implications. Ann Thorac Surg 1998; 65: 1529-1534.

5. Chuang SC, Hashibe M, Scelo G, et al. Risk of second primary cancer among esophageal cancer patients: A pooled analysis of 13 cancer registries. Cancer Epidemiol Biomarkers Prev 2008; 17: 1543-1549.

6. Slaughter DP, Southwick HW and Smejkal W. Field cancerization in oral stratified squamous epithelium; clinical implications of multicentric origin. Cancer 1953; 6: 963-968.

7. Abiko S, Shimizu Y, Miyamoto S, et al. Risk assessment of metachronous squamous cell carcinoma after endoscopic resection for esophageal carcinoma based on the genetic polymorphisms of alcoholdehydrogense-1B aldehyde dehydrogenase-2: Temperance reduces the risk. J Gastroenterol 2018; 53: 1120-1130.

8. Henry MA, Lerco MM, Naresse LE, et al. Outcome of superficial squamous cell carcinoma of the esophagus: A clinicopathological study. Acta Cir Bras 2013; 28: 373-378.

9. Alsop BR and Sharma P. Esophageal cancer. Gastroenterol Clin North Am 2016; 45: 399-412.

10. Lo OS, Law S, Wei WI, et al. Esophageal cancers with synchronous or antecedent head and neck cancers: A more formidable challenge? Ann Surg Oncol 2008; 15 : 1750-1756.

11. Berger A, Rahmi G, Perrod G, et al. Long-term followup after endoscopic resection for superficial esophageal squamous cell carcinoma: A multicenter Western study. Endoscopy 2019; 51: 298-306.

12. Kondo H, Fukuda H, Ono H, et al. Sodium thiosulfate solution spray for relief of irritation caused by Lugol's stain in chromoendoscopy. Gastrointest Endosc 2001; 53: 199-202.

13. Nonaka S, Saito Y, Oda I, et al. Narrow-band imaging endoscopy with magnification is useful for detecting metachronous superficial pharyngeal cancer in patients with esophageal squamous cell carcinoma. J Gastroenterol Hepatol 2010; 25: 264-269.

14. Miyazaki T, Sohda M, Higuchi T, et al. Effectiveness of FDG-PET in screening of synchronous cancer of other organs in patients with esophageal cancer. Anticancer Res 2014; 34: 283-287.

15. Lordick F, Mariette C, Haustermans $\mathrm{K}$, et al. Oesophageal cancer: ESMO Clinical Practice Guidelines for diagnosis, treatment and follow-up. Ann Oncol 2016; 27(suppl 5): v50-v57. 
16. Kuwano H, Nishimura Y, Oyama T, et al. Guidelines for diagnosis and treatment of carcinoma of the esophagus April 2012 edited by the Japan Esophageal Society. Esophagus 2015; 12: 1-30.

17. Bugter O, van de Ven SEM, Hardillo JA, et al. Early detection of esophageal second primary tumors using Lugol chromoendoscopy in patients with head and neck cancer: A systematic review and meta-analysis. Head Neck 2019; 41: 1122-1130.

18. Slim K, Nini E, Forestier D, et al. Methodological Index for Non-Randomized Studies (MINORS): Development and validation of a new instrument. ANZ J Surg 2003; 73: 712-716.

19. Moher D, Liberati A, Tetzlaff J, et al. Preferred Reporting Items for Systematic Reviews and MetaAnalyses: The PRISMA statement. J Clin Epidemiol 2009; 62: 1006-1012.

20. Muto M, Minashi K, Yano T, et al. Early detection of superficial squamous cell carcinoma in the head and neck region and esophagus by narrow band imaging: A multicenter randomized controlled trial. J Clin Oncol 2010; 28 : 1566-1572.

21. Schlemper RJ, Riddell RH, Kato Y, et al. The Vienna classification of gastrointestinal epithelial neoplasia. Gut 2000; 47: 251-255.

22. Higgins JP, Thompson SG, Deeks JJ, et al. Measuring inconsistency in meta-analyses. BMJ 2003; 327: 557-560.

23. Morimoto H, Yano T, Yoda Y, et al. Clinical impact of surveillance for head and neck cancer in patients with esophageal squamous cell carcinoma. World $J$ Gastroenterol 2017; 23: 1051-1058.

24. Shimizu Y, Tsukagoshi H, Fujita M, et al. Head and neck cancer arising after endoscopic mucosal resection for squamous cell carcinoma of the esophagus. Endoscopy 2003; 35: 322-326.

25. Motoyama S, Saito R, Kitamura M, et al. Outcomes of active operation during intensive followup for second primary malignancy after esophagectomy for thoracic squamous cell esophageal carcinoma. J Am Coll Surg 2003; 197: 914-920.

26. Kaneko K, Yano T, Minashi K, et al. Treatment strategy for superficial pharyngeal squamous cell carcinoma synchronously combined with esophageal cancer. Oncology 2013; 84: 57-64.

27. Kagei K, Hosokawa M, Shirato H, et al. Efficacy of intense screening and treatment for synchronous second primary cancers in patients with esophageal cancer. Jpn J Clin Oncol 2002; 32: 120-127.

28. Watanabe A, Hosokawa M, Taniguchi M, et al. Head and neck cancer associated with esophageal cancer. Auris Nasus Larynx 2007; 34: 207-211.

29. Onochi K, Shiga H, Takahashi S, et al. Risk factors linking esophageal squamous cell carcinoma with head and neck cancer or gastric cancer. J Clin Gastroenterol 2019; 53: e164-e170.

30. Katada C, Muto M, Nakayama M, et al. Risk of superficial squamous cell carcinoma developing in the head and neck region in patients with esophageal squamous cell carcinoma. Laryngoscope 2012; 122: 1291-1296.

31. Ioka A, Tsukuma H, Ajiki W, et al. Trends in head and neck cancer incidence in Japan during 1965-1999. Jpn J Clin Oncol 2005; 35: 45-47.

32. Yokokawa Y, Ohta S, Hou J, et al. Ecological study on the risks of esophageal cancer in $\mathrm{Ci}-\mathrm{Xian}$, China: The importance of nutritional status and the use of well water. Int J Cancer 1999; 83: 620-624.

33. Mourad M, Jetmore T, Jategaonkar AA, et al. Epidemiological trends of head and neck cancer in the United States: A SEER population study. J Oral Maxillofac Surg 2017; 75: 2562-2572.

34. Saeki H, Toh Y, Morita M, et al. The treatment outcomes of synchronous and metachronous esophageal squamous cell carcinoma and head and neck squamous cell carcinoma. Esophagus 2012; 9: 158-164.

35. Amar A, Franzi SA and Rapoport A. Evolution of patients with squamous cell carcinoma of upper aerodigestive tract. Sao Paulo Med J 2003; 121: 155-158. 